\title{
Prevalência de dor crônica em acadêmicos de uma Instituição de Ensino Superior
}

\section{Prevalence of chronic pain in academics of a Higher Education Institution}

\author{
Ramon Martins Barbosa, Renata Ferreira de Moura, Raisle \\ Vitoria Lima de Sousa, Bruno Santiago Silva, Jefferson Petto, Alan Carlos Nery dos \\ Santos
}

Como citar este artigo:

BARBOSA, RAMON M.; DE MOURA,

RENATA F.; DE SOUSA, RAISLE

V. L.; SILVA, BRUNO S.; PETTO,

JEFFERSON; DOS SANTOS, ALAN C.

N.; Prevalência de dor crônica em

acadêmicos de uma Instituição

de Ensino Superior. Revista Saúde

(Sta. Maria). 2020; 46 (2).

\section{Autor correspondente:}

Nome: Ramon Martins Barbosa

E-mail: ramonmartinsbarbosa@

hotmail.com

Telefone: (75) 99219889

Formação Profissional: Graduando em Fisioterapia pela Universidade Salvador (UNIFACS), Bahia, Brasil.

Filiação Institucional: Universidade Salvador - UNIFACS

Endereço para correspondência:

Rua: Av. Cetúlio Vargas, N²734

Bairro: Parque Getúlio Vargas

Cidade: Feira de Santana

Estado: Bahia

CEP: 44075-525

Data de Submissão:

06/03/2020

Data de aceite:

23/07/2020

Conflito de Interesse: Não há conflito de interesse

\section{(cc) $\mathrm{B} Y-\mathrm{NC}-\mathrm{ND}$}

\section{RESUMO}

Objetivo: Descrever a prevalência e fatores relacionados a dor crônica em universitários da área da saúde de uma instituição privada de ensino superior. Métodos: Estudo descritivo transversal com acadêmicos de ambos os sexos, dos cursos da saúde de uma universidade privada, localizada na cidade de Feira de Santana/BA. Todos os participantes foram submetidos a um questionário para coleta de dados sociodemográfica e o questionário adaptado a partir do PainDetectQuestionaire, para identificar indivíduos com dor crônica. Estudo aprovado pelo comitê de ética e pesquisa com CAE: 38987714.5.0000.5033. Os resultados foram descritos em valores absolutos e porcentagem. Resultados: Foram obtidas 306 respostas de 1.192 voluntários elegíveis. Desses, $77 \%$ mulheres, idade média $22 \pm 6$ anos, autodeclarados pardos (55\%) e negros (27\%). Os principais cursos de origem foram, a Fisioterapia (47\%) e a Farmácia (15\%). No tocante a dor, $62 \%$ relataram dor atual, enquanto, $18 \%$ tinham dor persistente. As regiões de maior queixa foram a lombar com $44 \%$, cabeça (frontal e temporal) $41 \%$, cervical $18 \%$ e membros inferiores $40 \%$. $52 \%$ dos voluntários com dor relataram atividade física regular. Conclusão: Os resultados indicam prevalência de dor atual em $62 \%$ dos universitários onde, $18 \%$ deles tem dor crônica. 0 tronco e a cabeça, foram identificados como os principais sítios de dor.

PALAVRAS-CHAVE: Dor crônica; Saúde Pública; Saúde do Estudante; Estudantes.

\section{ABSTRACT}

Objective: To describe the prevalence and factors related to chronic pain in university students in the health area of a private higher education institution. Methods: Descriptive cross-sectional study with academics of both sexes, from the health courses of a private university, located in the city of Feira de Santana/BA. All participants were submitted to a questionnaire to collect sociodemographic data and the questionnaire was adapted from the Pain Detect Questionaire, to identify individuals with chronic pain. Study approved by the ethics and research committee with CAE: 38987714.5.0000.5033. The results were described in absolute values and percentage. Results: 306 responses were obtained from 1,192 eligible volunteers. Of these, $77 \%$ women, mean age $22 \pm 6$ years, self-declared brown $(55 \%)$ and black $(27 \%)$. The main courses of origin were Physiotherapy (47\%) and Pharmacy (15\%). Regarding pain, $62 \%$ reported current pain, while $18 \%$ had persistent pain. The regions of greatest complaint were the lumbar region with $44 \%$, head (frontal and temporal) $41 \%$, cervical $18 \%$ and lower limbs $40 \% .52 \%$ of volunteers in pain reported regular physical activity. Conclusion: The results indicate a prevalence of current pain in $62 \%$ of university students, where $18 \%$ of them have chronic pain. The trunk and head were identified as the main pain sites.

KEYWORDS: Chronic pain; Public health; Student Health; Students. 


\section{INTRODUÇÃO}

A dor é definida como "uma experiência sensorial e emocional desagradável associada a dano tecidual real, potencial ou descrito em termos de tal dano". Sendo, considerada como o quinto sinal vital, a dor é caracterizada como uma percepção complexa influenciada pela associação de fatores físicos negativos e estado emocional ${ }^{1-2}$. Quando aguda, possui um valor biológico de preservação da integridade do indivíduo, pois serve como alerta para ocorrências de lesões no corpo ${ }^{3}$. Quando evoluída para o estado crônico, torna-se um problema grave, sendo causador de morbidade, absenteísmo ao trabalho e incapacidade temporária ou permanente ${ }^{4-5}$.

Além disso, a dor é um problema de saúde pública mundial. Ela acarreta de forma direta ou indireta em altos custos para os órgãos de saúde, cabendo ainda mencionar seus impactos negativos sobre aspectos biopsicossociais, e funcionas da população acometida por tal condição clínica ${ }^{6}$. Além disso, outro dado que coaduna com os impactos negativos impostos pela dor, é que, mais da metade dos pacientes que procuram pelos serviços de saúde apresentam a dor como sintoma principal sendo então considerada um fator limitante, que pode acarretar declínio na saúdee preocupação por parte da população ${ }^{7}$.

Evidências sugerem que acadêmicos em subgrupos específicos da população sofrem com dor crônica persistente. Tal acometimento vem sendo descrito principalmente em indivíduos do sexo feminino, onde a região da coluna lombar e cabeçaé evidenciada como os principais sítios de dor ${ }^{3,8-10}$. Esses dados são preocupantes, tendo em vista que, a dor tem impactos significativos sobre a funcionalidade durante as atividades de vida diária, laborais e, qualidade de vida do indivíduo afetado por tal condição clínica ${ }^{11-12}$.

Sendo assim, levando-se em conta a escassez de estudos que visem monitorar os níveis de dor dos futuros profissionais de saúde, bem como, a identificação de fatores que possam estar relacionados aos aspectos álgicos dos indivíduos, o presente estudo objetivou descrever a prevalência da dor crônica em acadêmicos de uma instituição de ensino superior privada.

\section{MÉTODO}

Estudo realizado a partir do projeto de pesquisa: "Avaliação do conhecimento sobre dor por parte dos discentes concluintes de Fisioterapia da cidade de Salvador", desenvolvido em uma instituição de ensino superior privada, localizada na cidade de Salvador - BA. A pesquisa foi submetida e aprovada pelo comité de ética e pesquisa da referida instituição sobre parecer no 106938/2014 e CAAE: 38987714.5.0000.5033.

O presente estudo utilizou-se de uma análise descritiva transversal para avaliar a prevalência e fatores relacionados a dor crônica em acadêmicos de uma instituição de ensino superior privada. Foram incluídos estudantes de 
ambos os sexos, regularmente matriculados nos cursos da área de saúde de uma instituição de ensino superior privada da cidade de Feira de Santana - BA, com idade entre 18 e 60 anos. Sendo excluídos, acadêmicos que tiveram doenças crônicas e degenerativas diagnosticadas e/ou, em processo de investigação, lesões agudas, tratadas ou, em fase de tratamento.

A coleta dos dados ocorreu entre agosto de 2018 e março de 2019. Os participantes foram sequencialmente reunidos em suas respectivas salas de aulas, onde cada um recebeu o material a ser respondido, com instruções e recomendações de preenchimento diretamente pelos responsáveis da pesquisa, estando presentes no local para esclarecimento de possíveis dúvidas. Não foi estipulado limite de tempo para a conclusão do preenchimento dos questionários, nem permitido contato entre os estudantes para que as respostas não sofressem interferências.Foram utilizados dois instrumentos para recolhimento dos dados. Em primeiro momento, foi utilizado um questionário social criado pelos pesquisadores, constituído por perguntas sobre: características sociodemográficas (sexo, idade, etnia e condição socioeconômica), comportamento psicossocial (fumo e álcool) e atividade/exercício físico.

Já para análise da dor e dos fatores a ela relacionados, utilizou-se um questionário adaptado a partir do PainDetectQuestionairie. Assim, foram removidas as informações referentes da dor neuropática e inseridas questões para identificar a presença de dor crônica. A ferramenta final consistiu das perguntas: nas últimas semanas você sente/ sentiu dor nos ossos, músculos ou articulações em repouso? Por favor, indique se sente dor a mais de 3 meses, ou menos que 3 meses. Como você avalia sua dor neste momento? Qual a intensidade da dor mais forte, nas últimas 4 semanas? Qual a intensidade da dor mais forte, nas últimas 4 semanas, em média? Essas perguntas eram acompanhadas de uma escala numérica de Zero a Dez pontos (Zero=sem dor, Dez=grave/dor máxima) que representa a intensidade da percepção da dor. A segunda seção inclui um diagrama corporal, na vista anterior e posterior, no qual os participantes deveriam realizar uma pintura, indicando as regiões em que sentia dor. Por fim, a classificação da dor em aguda ou crônica foi realizada com base nas recomendações da Classificação Internacional de Doenças - CID-11, onde define que a dor crônica é uma dor persistente ou recorrente superior a três 3 meses ${ }^{13}$.

Toda a pesquisa foi realizada de acordo com as recomendações da Declaração de Helsinque e a Resolução CNS 466/12. Todos os participantes assinaram o Termo de Consentimento Livre e Esclarecido. Os dados obtidos foram calculados, e tabulados em planilhas do Microsoft Excel, sendo descritos posteriormente de forma absoluta, média e em porcentagem.

Para facilitar o entendimento dos resultados e a análise dos dados, os itens do questionário foram agrupados em características sociodemográficas, aspectos relacionados a dor e classificação/intensidade da dor. 


\section{RESULTADO}

Tabela 1. Características sociodemográficas dos universitários de uma instituição de ensino superior privada no município de Feira de Santana - BA.

\begin{tabular}{|c|c|c|}
\hline Variável & $(\mathrm{N}=306)$ & $\%$ \\
\hline Idade & $22 \pm 6$ & \\
\hline \multicolumn{3}{|l|}{ Gênero } \\
\hline$F$ & 238 & 77,78 \\
\hline M & 68 & 22,22 \\
\hline \multicolumn{3}{|l|}{ Raça } \\
\hline Branco & 54 & 17,65 \\
\hline Negro & 84 & 27,45 \\
\hline Pardo & 170 & 55,56 \\
\hline Outros & 8 & 2,61 \\
\hline \multicolumn{3}{|l|}{ Estado civil } \\
\hline Solteiro & 269 & 87,91 \\
\hline Casado & 33 & 10,46 \\
\hline Divorciado & 4 & 1,31 \\
\hline \multicolumn{3}{|l|}{ Curso de Origem } \\
\hline Biomedicina & 14 & 4,58 \\
\hline Educação Física & 15 & 4,90 \\
\hline Enfermagem & 42 & 13,73 \\
\hline Farmácia & 46 & 15,03 \\
\hline Fisioterapia & 146 & 47,71 \\
\hline Nutrição & 23 & 7,52 \\
\hline Psicologia & 31 & 10,13 \\
\hline \multicolumn{3}{|c|}{ Situação de emprego } \\
\hline Trabalha & 101 & 33,01 \\
\hline Não trabalha & 205 & 66,99 \\
\hline \multicolumn{3}{|l|}{ Renda } \\
\hline Sem renda & 142 & 46,41 \\
\hline <1 salário & 58 & 18,95 \\
\hline 1 salário & 64 & 20,92 \\
\hline 2 salários & 28 & 9,15 \\
\hline 3 salários & 42 & 13,93 \\
\hline \multicolumn{3}{|l|}{ Álcool } \\
\hline S & 140 & 45,80 \\
\hline $\mathrm{N}$ & 166 & 54,20 \\
\hline
\end{tabular}




\begin{tabular}{lll} 
Tabagismo & & \\
$\mathrm{S}$ & 5 & 1,63 \\
$\mathrm{~N}$ & 301 & 98,37 \\
Atividade/Exercício físico & & \\
$\mathrm{S}$ & 101 & 33,01 \\
$\mathrm{~N}$ & 205 & 66,91 \\
\hline F= Feminino; M= Masculino; <= Menor que; $S=$ Sim; $N=$ Não.
\end{tabular}

Dos 1.192 acadêmicos, 306 responderam aos questionários que compuseram a amostra desta pesquisa. A média de idade dos participantes foi de $22 \pm 6$ anos, havendo predominância do sexo feminino $(77,7 \%)$, além disso, a maior parcela da amostra constitui-sede indivíduos autodeclarados com cor de pele parda (55,5\%). Os solteiros, representaram (87,91\%), 66,9\% afirmaram não trabalhar e 46,41\% não obter renda. Os cursos que mais estiveram presentes nas respostas foram a Fisioterapia (47,7\%), Farmácia (15\%), Enfermagem (13,7\%) e Psicologia (10,1\%), Tabela 1.

Tabela 2. Aspectos relacionados a dor em universitários de uma instituição de ensino superior privada no município de Feira de Santana - BA.

\begin{tabular}{lll}
\hline Variável & $(\mathbf{n}=)$ & $\%$ \\
\hline Presença de dor & & \\
S & 191 & 62,4 \\
N & 115 & 37,6 \\
Dor persistente & & \\
S & 57 & 18,1 \\
N & 249 & 81,9 \\
Atividade/exercício físico & & \\
S & 99 & 52 \\
N & 92 & 48 \\
Regiões de maior quadro álgico & & \\
Lombar & 134 & 44,26 \\
Cabeça (Frontal / Temporal) & 126 & 41,61 \\
MMll & 121 & 40,01 \\
Cervical & 54 & 18,13 \\
Torácica & 43 & 14,1 \\
Ombro & 43 & 14,1 \\
Abdome & 31 & 10,10 \\
Braço & 20 & 6,51 \\
Mão & 15 & 4,6 \\
& &
\end{tabular}




\begin{tabular}{lll} 
Antebraço & 7 & 2,32 \\
Seio & 3 & 0,9 \\
Região Pélvica & 2 & 0,6 \\
Orelhas & 2 & 0,6 \\
Cotovelo & 1 & 0,3 \\
\hline
\end{tabular}

$S=\operatorname{Sim} ;$ N= Não; MMII= Membros Inferiores.

A Tabela 2 apresenta a distribuição das respostas relacionadas aos aspectos relacionados a dor dos universitários. Nela observamos que,a maioria dos estudantes tinham dor no momento da avaliação (62\%) enquanto, 18\% apresentavam dor de característica crônica. Assim, as regiões corporais de maior acometimento foram a cabeça (frontal e temporal) $41 \%$, lombar $44 \%$, membros inferiores $40 \%$, cervical $18 \%$, torácica $14,1 \%$ e ombro $14,1 \%$. Sendo que, $52 \%$ dos voluntários com dor relataram praticar atividade/exercício física regularmente.

Já na Tabela 3 ainda em relação aos aspectos da dor, o dado mais expressivo é que quando questionados sobre à intensidade média da dor mais forte nas últimas 4 semanas, mesmo relatando anteriormente sentir dor, os voluntários assinalaram, Zero $(5,57 \pm 2,62)$, Leve $(5,58 \pm 2,62)$, Moderada $(5,65 \pm 2,59)$ e Grave $(5,58 \pm 2,60)$.

Tabela 3. Classificação e intensidade da dor em universitários de uma instituição de ensino superior privada no município de Feira de Santana - BA.

\begin{tabular}{|c|c|c|c|}
\hline \multicolumn{4}{|c|}{ Classificação da dor atual } \\
\hline Zero & Leve & Moderada & Grave \\
\hline 50,4 & 35,24 & 13,38 & 0,98 \\
\hline \multicolumn{4}{|c|}{ Intensidade média da dor nas últimas 4 semanas } \\
\hline Zero & Leve & Moderada & Grave \\
\hline $4,49 \pm 2,21$ & $4,50 \pm 2,21$ & $4,55 \pm 2,20$ & $4,63 \pm 2,30$ \\
\hline \multicolumn{4}{|c|}{ Intensidade média da dor mais forte nas últimas 4 semanas } \\
\hline Zero & Leve & Moderada & Grave \\
\hline $5,57 \pm 2,62$ & $5,58 \pm 2,62$ & $5,65 \pm 2,59$ & $5,58 \pm 2,60$ \\
\hline
\end{tabular}

\section{DISCUSSÃO}

Em resposta aos objetivos deste estudo, identificamos alta prevalência de dor entre os universitários dos cursos de saúde, sendo que 18\% deles tinham dor crônica persistente. As regiões com maior frequência de queixas álgicas foram a lombar, cabeça, membros inferiores, cervical e torácica respectivamente. Além disso, chama atenção, que 
mesmo informando ter dor nas últimas quatro semanas, os voluntários referiram no momento da avaliação dor zero, leve, moderada e grave.

Embora poucos estudos tenham se dedicado a estudar dor em universitários de saúde, os resultados encontrados suscitam preocupação, isso porque, em teoria, populações com maior grau de instrução apresentariam maior capacidade de auto-gestão da dor e, consequentemente, menor prevalência de cronificação dos sintomas ${ }^{14-15}$. Contudo, assim como o nosso estudo, outras pesquisas têm apontado dados alarmantes. De fato, Silva et al. ${ }^{3}$ identificaram uma prevalência de dor crônica de 59,7\%, em acadêmicos de Enfermagem. Embora esse achado esteja em linha com observações que apontam prevalência de dor crônica no Brasil, que varia entre 29,3 a 73,3\%, esses dados podem não refletir a realidade da população estudada, uma vez que, a dor pode ser influenciada por diversos fatores, incluindo a regionalização ${ }^{16-17}$.

Por outro lado, além da prevalência geral, também podemos notar altos índices de dor em regiões anatômicas específicas, destacando-se entre elas a dor lombar, as dores na cabeça e nos membros inferiores. Corroborando com esses achados, outros estudos reportaram elevada prevalência de lombalgia em universitários ${ }^{8-10}$. De fato, Silva et al. ${ }^{9}$ identificaram uma prevalência de 35,6\% de dor na região lombar em acadêmicos do curso de medicina, chamando atenção o fato de que, mesmo em uma população relativamente jovem, há uma alta prevalência deste sintoma. Outro dado que coaduna é que, se levarmos em consideração que boa parte da população mundial tem, teve ou terá a sintomatologia da dor lombar, boa parte destes jovens acadêmicos já se adentram a esta estimativa 8,18 .

Neste sentido, com relação a frequência de dorlombar, existem crescentesteorias para explicar a dor na região. Podendo ser relacionada com hipóteses de esfera emocional,visceral e somática ${ }^{19}$. Com relação a esfera emocional, Sribastav et al. ${ }^{20}$ identificaram que, em pacientes com dor lombar existe uma maior incidência de ansiedade e depressão, quando comparada a pessoas saudáveis, demonstrando um dado preocupante, tendo em vista que, a ansiedade e depressão é uma realidade entre os universitários ${ }^{21}$. Outra hipótese também é que,a alteração dos músculos inspiratórios possa estar envolvida na gênese da dor lombar ${ }^{22}$. Tendo em vista que, o diafragma desempenha um papel fundamental na postura lombar, e seu declínio funcional poderá acarretar em dor na região ${ }^{23}$. Entretanto, embora os mecanismos que justifiquem tais resultados não estejam claros, é preciso lembrar que a maioria dos casos de dor lombar é de origem inespecífica ${ }^{24}$.

Somado aos dados já apresentados, nossos resultados indicam baixa percepção dolorosa na população avaliada. Esse resultado pode se justificar no fato de que se trata de um público com elevado grau de instrução, além disso, mais de 50\% dos avaliados praticam atividade física regular. Em conjunto, educação e prática regular de atividade física são apontados como fatores que podem aumentar a resiliência a dor, além de minimizar as complicações e potencializar 0 tratamento desse fenômeno clínico biopsicossocial ${ }^{14-15,25}$.

Algumas limitações no presente estudo devem ser consideradas. Embora os resultados deste estudo sejam 
importantes para direcionar novas perspectivas na saúde, o desenho transversal do estudo, não permite uma adequada relação de causalidade. Além disso, uma amostra unicêntrica limita a extrapolação dos dados, principalmente por não incluir universidades públicas e outras instituições de ensino superior da mesma região. Entretanto, estudos em outros centros privados identificaram prevalência semelhante s.9. $^{3,9}$

Por fim, os resultados apresentados apontam para um dado preocupante. Pois, está relacionada com elevados impactos financeiros, bem como, afastamento de atividades laborativas e sociais. Desta forma, carecendo de atenção da universidade, demais instituições de ensino superior e órgãos públicos de saúde. Tendo em vista que, os dados supracitados refletem a realidade dos futuros profissionais das ciências da saúde.

\section{CONSIDERAÇÕES FINAIS}

Em síntese, os achados deste estudo indicam alta prevalência de dor crônica entre os acadêmicos das ciências da saúde, sendo a região lombar, cabeça e membros inferiores apontadas como os principais sítios de dor. Também identificamos baixa percepção dolorosa por parte dos acadêmicos. Além disso, mais da metade dos acadêmicos com dor informaram praticar atividade física regular. Podendo implicar em melhorias para indivíduos com dor, tendo em vista que, a atividade física regular é apontada como um fator determinante que pode aumentar a resiliência a dor.

\section{REFERÊNCIAS}

1. Araújo LC, Romero B. Dor: avaliação do quinto sinal vital: Uma reflexão teórica. Rev Dor. 2015; 16(4): 291-6.doi: 10.5935/1806-0013.20150060

2. Nascimento LA, Cardoso MG, Oliveira SA, Quina E, Sardinha DS. Manuseio da dor: avaliação das práticas utilizadas por profissionais assistenciais de hospital público secundário. Rev Dor. 2016; 17(2): 76-80.doi: 10.5935/1806-0013.20160019.

3. Silva CD, Ferraz GC, Souza LAF, Cruz LVS, Stival MM, Pereira LV. Prevalence of chronic pain in nursing undergraduate students. Texto e Context Enferm. 2011;20(3):319-25. doi: 10.1590/S0104-07072011000300013

4. Silva KN, Dutra FCMS. Fatores psicossociais do trabalho e dor crônica: análise em duas escolas da rede municipal de educação em Serrana/SP. Rev Dor. 2016; 17(3):164-70.doi: 10.5935/1806-0013.20160064. 
5. Carvalho RC, Maglione CB, Machado GB, Araújo JE, Silva JRT, Silva ML. Prevalenceandcharacteristicsofchronicpain in Brazil: a national internet-basedsurveystudy. Br J Pain. 2018; 1(4):331-8.doi: 10.5935/25950118.20180063

6. Pereira FG, França MH, Paiva MCA, Andrade LH, Viana MC. Prevalenceandclinical profile ofchronicpainand its associationwith mental disorders. Rev Saúde Pública. 2017;51: 96.doi: 10.11606/s1518-8787.2017051007025

7. Santos ACN, Barbosa ML, Souza AG, Petto J. Conhecimento dos acadêmicos e profissionais da fisioterapia sobre dor: uma revisão sistemática. ABCS Health. 2015;42(2):99-104. doi:10.7322/abcshs.v42i2.1010.

8. Alfieri FM, Oliveira NC, Santana IEFC, Ferreira KMP, Pedro RDM. Prevalência de dor lombar em universitários da saúde e sua relação com estilo de vida e nível de atividade física. RevInspir. 2016;11(4):27-31.

9. Silva AL, Smaidi K, Pires MHR, Pires OC. Prevalence of chronic pain and associated factors among medical students. Rev Dor. 2017;18(2):108-11. doi: 10.5935/1806-0013.20170022.

10. Morais ML, Silva VKO, Silva JMN. Prevalenceoflowbackpainandassociatedfactorsamongphysiotherapystudents. Br J Pain. 2018;1(3):241-27.doi: 10.5935/2595-0118.20180047

11. Silva GG, Sirena SA. Perfil de encaminhamentos a fisioterapia por um serviço de Atenção Primária à Saúde, 2012. EpidemiolServ Saúde. 2015;24(1):123-33.doi: 10.5123/S1679-49742015000100014.

12. Santos ACN, Bahiano LAB, Barbosa RM, Barbosa ML, Souza AG, Petto J. Conhecimento dos acadêmicos de fisioterapia sobre o manejo da dor. Fisioter Bras. 2019;20(3):369-75.doi: 10.33233/fb.v20i3.2783

13. Aziz Q, Barke A, Bennett MI, Benoliel R, Cohen M, Evers S, et al. A classification of chronic pain for ICD11. Pain. 2015;156(6):1003-7.

14. Pardo GB, Girbés EL, Roussel NA, Izquierdo TG, Penick VJ, Martín DP. Pain Neurophysiology Education and Therapeutic Exercise for Patients With Chronic Low Back Pain: A Single-Blind Randomized Controlled Trial. Arch Phys Med Rehabil. 2018;99(2):338-47. doi: 10.1016/j.apmr.2017.10.016. 
15. Moseley L. Combined physiotherapy and education is efficacious for chronic low back pain. Aust J Physiother. 2002;48(4):297-302. doi: 10.1016/s0004-9514(14)60169-0

16. Nascimento PRC, Costa LOP. Prevalência de dor lombar no Brasil: Uma revisão sistemática. Cad. Saúde Pública. 2015;31(6):1141-1155. doi: 10.1590/0102-311X00046114

17. Vasconcelos FH, Araújo GC. Prevalência de dor crônica no Brasil: estudo descritivo. Br J Pain. 2018;1(2):176-9.doi: 10.5935/2595-0118.20180034

18. Hoy D, Brooks P, Blyth F, Buchbinder R. The Epidemiologyoflowbackpain. Best Pract Res ClinRheumatol. 2010;24(6):769-81. doi: 10.1016/j.berh.2010.10.002

19. Bordoni B, Marelli F. Morabito B. Saconni B. Caiazzo P. Castagna R. Lowbackpainandgastroesophagealreflux in patientswith COPD: thedisease in thebreath. InternationalJournalof COPD 2018;13(1):325-334.doi: 10.2147/ COPD.S150401

20. Sribastav SS, Peiheng H, Jun L, zemin L, Fuxin W, Jianru W, et al. Interplayamongpainintensity, sleepdisturbanceandemotion in patientswith non-specificlowbackpain. PeerJ. 2017:5:e3282:1-18. doi: 10.7717/peerj.3282

21. Rotenstein LS, Ramos MA, Torre M, et al. Prevalenceofdepression, depressivesymptoms, andsuicidalideationamong medical students: a systematic review and meta-analysis. JAMA. 2016;316(21):2214-2236.doi: 10.1001/ jama.2016.17324

22. Ostwal PP, Wani SK. Breathingpatterns in patientswithlowbackpain. Int J Physiother Res 2014;2(1):34753.

23. Beeckmans $\mathrm{N}$, Vermeersch A, Lysens $\mathrm{R}$, et al. The presenceofrespiratorydisorders in individualswithlowbackpain: a systematic review. Man Ther. 2016;26:77-86.doi: 10.1016/j.math.2016.07.011

24. Oliveira CB, Maher CG, Pinto RZ, Traegér AC, Lin CWC, Chenot JF, et al. Clinical practice guidelines for the management of non-specific low back pain in primary care: an updated overview. 2018;27(11):2791-2896.doi: 
10.1007/s00586-018-5786-7.

25. Cunha CO, Pinto-Fiamengui LM, Sampaio FA and Conti PC. Is aerobic exercise useful to manage chronic pain? Rev Dor. 2016;17(1):61-4.doi: 10.5935/1806-0013.20160015 